\title{
Immune profiling and immunotherapeutic targets in pancreatic cancer
}

\author{
Felicia L. Lenzo ${ }^{1}$, Shumei Kato ${ }^{2}$, Sarabjot Pabla ${ }^{1}$, Paul DePietro ${ }^{1}$, Mary K. Nesline ${ }^{1}$, Jeffrey M. Conroy ${ }^{1,3}$, \\ Blake Burgher $^{1}$, Sean T. Glenn ${ }^{1,3,4}$, Boris Kuvshinoff ${ }^{5}$, Razelle Kurzrock ${ }^{2}$, Carl Morrison ${ }^{1,3,6,7}$ \\ ${ }^{1}$ OmniSeq, Inc., Buffalo, NY, USA; ${ }^{2}$ Center for Personalized Cancer Therapy, Moores Cancer Center, La Jolla, CA, USA; ${ }^{3}$ Center for Personalized \\ Medicine, Roswell Park Comprehensive Cancer Center, Buffalo, NY, USA; ${ }^{4}$ Department of Molecular and Cellular Biology, Roswell Park \\ Comprehensive Cancer Center, Buffalo, NY, USA; ${ }^{5}$ Department of Surgery, Roswell Park Comprehensive Cancer Center, Buffalo, NY, USA; \\ ${ }^{6}$ Department of Pathology, Roswell Park Comprehensive Cancer Center, Buffalo, NY, USA; ${ }^{7}$ Cancer Genetics and Genomics, Roswell Park \\ Comprehensive Cancer Center, Buffalo, NY, USA \\ Contributions: (I) Conception and design: All authors; (II) Administrative support: All authors; (III) Provision of study materials or patients: All \\ authors; (IV) Collection and assembly of data: All authors; (V) Data analysis and interpretation: All authors; (VI) Manuscript writing: All authors; (VII) \\ Final approval of manuscript: All authors. \\ Correspondence to: Carl Morrison. Roswell Park Comprehensive Cancer Center, Elm \& Carlton Streets, Buffalo, NY 14263, USA. \\ Email: carl.morrison@omniseq.com.
}

Background: Immunotherapeutic approaches for pancreatic ductal adenocarcinoma (PDAC) are less successful as compared to many other tumor types. In this study, comprehensive immune profiling was performed in order to identify novel, potentially actionable targets for immunotherapy.

Methods: Formalin-fixed paraffin embedded (FFPE) specimens from 68 patients were evaluated for expression of 395 immune-related markers (RNA-seq), mutational burden by complete exon sequencing of 409 genes, PD-L1 expression by immunohistochemistry (IHC), pattern of tumor infiltrating lymphocytes (TILs) infiltration by CD8 IHC, and PD-L1/L2 copy number by fluorescent in situ hybridization (FISH).

Results: The seven classes of actionable genes capturing myeloid immunosuppression, metabolic immunosuppression, alternative checkpoint blockade, CTLA-4 immune checkpoint, immune infiltrate, and programmed cell death 1 (PD-1) axis immune checkpoint, discerned 5 unique clinically relevant immunosuppression expression profiles (from most to least common): (I) combined myeloid and metabolic immunosuppression [affecting 25 of 68 patients (36.8\%)], (II) multiple immunosuppressive mechanisms (29.4\%), (III) PD-L1 positive (20.6\%), (IV) highly inflamed PD-L1 negative (10.3\%); and (V) immune desert $(2.9 \%)$. The Wilcoxon rank-sum test was used to compare the PDAC cohort with a comparison cohort ( $n=1,416$ patients) for the mean expressions of the 409 genes evaluated. Multiple genes including TIM3, VISTA, CCL2, CCR2, TGFB1, CD73, and CD39 had significantly higher mean expression versus the comparison cohort, while three genes (LAG3, GITR, CD38) had significantly lower mean expression.

Conclusions: This study demonstrates that a clinically relevant unique profile of immune markers can be identified in PDAC and be used as a roadmap for personalized immunotherapeutic decision-making strategies.

Keywords: Precision immunotherapy; PD-L1; immune suppression; immune activation; checkpoint inhibitors

Submitted Jan 28, 2020. Accepted for publication Sep 29, 2020.

doi: $10.21037 /$ atm-20-1076

View this article at: http://dx.doi.org/10.21037/atm-20-1076 


\section{Introduction}

Data from recent clinical trials suggest that immune checkpoint inhibitors targeting cytotoxic T-lymphocyte associated protein 4 (CTLA4), programmed cell death 1 (PDCD1; best known as PD-1), or CD274 (best known as $\mathrm{PD}-\mathrm{L} 1$ ) are poorly effective as stand-alone therapeutic interventions in individuals with pancreatic cancer (1-3). The lack of efficacy of these immunotherapeutics may reflect the fact that pancreatic oncogenesis is generally accompanied by the establishment of a strongly immunosuppressive tumor microenvironment, as well as by a robust stromal reaction (the so-called "desmoplastic stroma") that is expected to counteract tumor infiltration by immune effector cells (4-6). Thus, pancreatic ductal adenocarcinoma (PDAC) are generally abundant in $\mathrm{CD}^{+} \mathrm{FOXP}^{+}$regulatory T-cells $\left(\mathrm{T}_{\mathrm{REG}}\right), \mathrm{M} 2-$ like tumor-associated macrophages (TAMs), myeloidderived suppressor cells (MDSCs), and $\mathrm{FAP}^{+}$cancerassociated fibroblasts, which together generate a local microenvironment that inhibits innate and adaptive immunity (7). Moreover, several proteins involved in metabolic immunosuppression, such as ectonucleoside triphosphate diphosphohydrolase 1 (ENTPD1; best known as CD39), an extracellular nucleotidase that precipitates the conversion of immunostimulatory ATP into immunosuppressive adenosine, as well as adenosine A2 a receptor (ADORA2A), which transduces such immunosuppressive effects, are overexpressed in the PDAC microenvironment (8-11).

A potential approach to treat patients with PDAC is to combine checkpoint inhibitors, chemotherapy, and/ or radiation therapy with small molecules designed to reverse immunosuppression beyond checkpoint blockade, potentially enabling $\mathrm{CD}^{+}$cytotoxic $\mathrm{T}$ lymphocyte (CTL)dependent tumor eradication $(12,13)$. Promising work in this area comes from a single institution Phase 2 clinical trial combining a C-C motif chemokine receptor 2 (CCR2) inhibitor with the FOLFIRINOX (folinic acid plus 5 -fluorouracil plus irinotecan plus oxaliplatin) regimen in patients with borderline resectable and locally advanced PDAC, resulting in local tumor control in $97 \%$ of cases $(\mathrm{n}=32)(14,15)$. This reflects the ability of C-C motif chemokine ligand 2 (CCL2) produced by PDAC cells to attract immunosuppressive CCR2 ${ }^{+}$TAMs, ultimately resulting in CTL exclusion and the establishment of a "cold tumor" microenvironment $(16,17)$. However, the true incidence of patients with PDAC that overexpress immunosuppressive cytokines including (but not limited to)
CCR2 is unknown. This constitutes an obvious obstacle to the design of novel therapeutic regimens aimed at reversing immunosuppression beyond checkpoint blockade for the treatment of PDAC.

Here, we demonstrate that comprehensive immune profiling, simultaneously assessing PD-L1 activation status by immunohistochemistry (IHC) and fluorescence in situ hybridization (FISH), CTL infiltration by IHC, tumor mutational burden (TMB), microsatellite instability (MSI), and the expression levels of immunological relevant transcripts can provide an initial overview of the immunological landscape in patients with PDAC with a particular focus on actionable pathways (18). The overall objective of this study is to generate awareness of which immunosuppressive pathways are likely to be activated in PDAC and enable the rational design of clinical trials targeting such pathways.

We present the following article in accordance with the MDAR reporting checklist (available at http://dx.doi. org/10.21037/atm-20-1076).

\section{Methods}

\section{Patients}

Formalin-fixed paraffin embedded (FFPE) blocks or slides from sixty-eight (68 PDAC patients (34 primary, 34 metastatic) were evaluated to identify actionable targets for immunomodulatory immunotherapeutic drugs using comprehensive immune profiling via Immune Report Card $^{\circledR}$ (IRC). IRC is a commercially available test approved for clinical use by the New York State Clinical Laboratory Evaluation Program (NYS CLEP). Testing was performed in a CLIA certified laboratory at OmniSeq, Inc. (Buffalo, $\mathrm{NY}$ ). An additional 1,416 non-PDAC tumor samples that also underwent IRC testing were used as the comparison cohort to determine which genes were differentially expressed within PDAC. All tumor samples were collected in the context of patient clinical care. Associated deidentified molecular data was considered non-human subjects research under IRB approved BDR \#073166 at Roswell Park Comprehensive Cancer Center (Buffalo, NY) and in accordance with the Helsinki declaration (as revised in 2013).

\section{$T M B$}

DNA was extracted from each sample and evaluated for TMB by whole-exon DNAseq as previously described 
with minor modifications $(19,20)$. TMB qualified, nonsynonymous somatic variants are reported as mutations per megabase (Mut/Mb). A high TMB cutoff was defined as the top tenth percentile of values ( 7 mutations per megabase of DNA or higher) observed in this study specific to PDAC (21). Cases with less than $20 \%$ neoplastic nuclei $(n=4)$ were excluded from the analysis.

\section{Immunobistochemical studies}

The expression of PD-L1 on the surface of tumor cells was assessed in all samples via the Dako Omnis platform (Agilent, Santa Clara, CA) using the anti-PD-L1 22C3 pharmDx antibody (Agilent, Santa Clara, CA), as per manufacturers protocol (22). Expression levels were scored as per published guidelines (23). Additional serially sectioned tissue was evaluated for lymphocyte infiltration using the anti-CD8 antibody C8/144B (Agilent, Santa Clara, CA) at a dilution ratio of 1:75 and assigned a qualitative score of non-infiltrated, infiltrated, or excluded. Non-infiltrated referred to a sparse number of $\mathrm{CD}^{+}{ }^{+} \mathrm{T}$-cells that infiltrate nests of neoplastic cells in a non-overlapping fashion and with less than $5 \%$ of the tumor showing an infiltrating pattern. Infiltrated represents frequent $\mathrm{CD} 8^{+} \mathrm{T}$-cells that infiltrate nests of neoplastic cells in an overlapping fashion at least focally and in more than $5 \%$ of the tumor. Excluded represents restriction of more than $95 \%$ of all $\mathrm{CD}^{+} \mathrm{T}$-cells in a tumor to the periphery or interstitial stromal areas and not actively invading nest or groups of neoplastic cells.

\section{PD-L1/2 copy number}

Fluorescence in situ hybridization (FISH) was performed as previously described (24). Briefly, locus specific bacterial artificial chromosomes (BAC) for PD-L1/2 at 9p24.1 were labeled with SpectrumOrange and a commercially available SpectrumGreen chromosome 9 centromeric probe (Abbott Molecular/Vysis, Des Plaines, Illinois) were hybridized to test samples and not less than 60 neoplastic cells were evaluated for copy number gain as defined by CAP ASCO (College of American Pathologists; American Society of Clinical Oncology) HER2 guidelines (25).

\section{Microsatellite instability (MSI)}

MSI analysis, for a subset of cases, was performed using polymerase chain reaction (PCR) amplification with detection of product size by capillary electrophoresis (GeneMapper software version 5.0) using fluorescentlylabeled primers for amplification of five markers including two mononucleotide repeat markers (BAT-25, BAT-26) and three dinucleotide repeat markers (D2S123, D5S346, and D17S250). MSI at $\geq 2$ loci was defined as MSI-H (high), instability at a single locus was defined as MSI-L (low), and no instability at any of the loci tested was defined as microsatellite stable (MSS). For the remaining cases, MSI analysis was performed using next generation sequencing of a panel targeting 29 homopolymer regions. Briefly, TruSeq Custom Amplicon libraries (Illumina) were generated to carry out multiplexed targeted sequencing of the 29 homopolymer loci, clustered and then sequenced on a MiSeq system (Illumina). A pipeline was created to read ".fastq" files and conduct sequence alignment, variant calling, and indel extraction to determine MSI or MSS status.

\section{Immune expression analysis}

Multiplexed gene-specific primer pairs and next generation sequencing were used to assess expression of 394 immunerelated genes (https://cdn.amegroups.cn/static/public/ ATM-20-1076-Supplementary.pdf) from RNA isolated from FFPE slides $(19,20)$. Transcript abundance was normalized and compared to an internal reference population encompassing 338 patients from twenty-nine solid tumor types. The reference population represented a broad dynamic range of expression values which were used to rank gene expression in test samples, as previously described (19). Rank values were set on a scale of 1 to 100 and stratified into "High" [85-100], "Moderately high" [75-84], "Moderate" [50-74], "Moderately low" [25-49], and "Low" [0-24].

\section{Statistical analysis}

The expression levels of 23 genes that code for targets of immunomodulatory immunotherapeutic agents currently in clinical development were used for precision immunotherapy evaluation (Table 1). Prevalence of gene expression rank for each actionable target was calculated as the mean percentage of PDAC cases that ranked moderately high, high, or very high (expression rank $\geq 75$ ) as compared to the ranks for all tumor types in the reference population. Wilcoxon Rank-Sum tests were performed to compare the mean expression ranks of unique genes in the pancreatic 
Table 1 Differential expression of immunotherapeutic targets

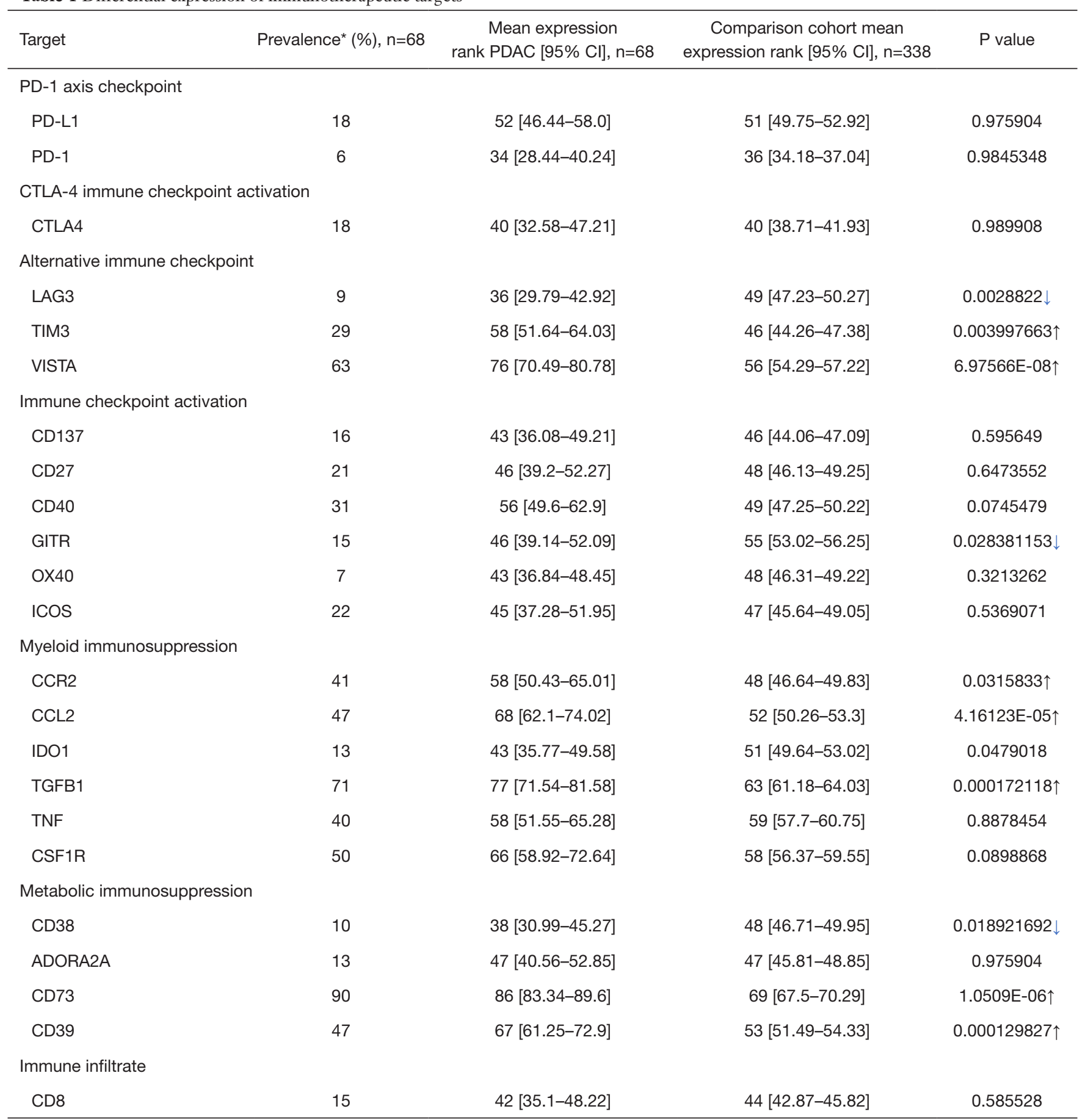

*, prevalence was the percent of patients with gene mean expression rank $\geq 75$ compared to the total cohort ( $n=68$ ); $\uparrow / \downarrow$, statistically significant expression ranks in pancreatic patients $(n=68)$ that were higher (red arrow) or lower (blue arrow) compared to the expression rank of the comparison cohort $(n=1,416)$. PDAC, pancreatic ductal adenocarcinoma. 
cohort $(n=68)$ to the remaining tested patients $(n=1,416)$ across all disease sites, statistical significance if $\mathrm{P}<0.05$.

\section{Association of therapy and clinical trials}

Publicly available sources of drug descriptions and gene associations with known aliases were queried to curate a database of immunotherapeutic agents and their targets.

\section{Clinically relevant immune gene expression profiles in PDAC}

Twenty-three key immune-related genes were divided into seven different classes of actionable genes based upon therapeutic approaches that included immune infiltrate (CD8, CD4, FOXP3, CD68, CD163), CTLA-4 immune checkpoint (CTLA-4), PD-1 axis immune checkpoint (PD-L1, PD-1, PD-L2), alternative immune checkpoint (VISTA, TIM3, LAG3), immune checkpoint activation (CD137, CD27, GITR, CD40, OX40, ICOS), myeloid immunosuppression (CCL2, CCR2, CSF1R, IDO1, IL10, TGFB1), and metabolic immunosuppression (ADORA2A, CD39, NT5E, CD38). Expression of these genes as compared to a comparison cohort expression database of multiple tumor types $(\mathrm{n}=1,416)$ was used to derive clinically relevant subtypes of PDAC that included combined myeloid metabolic immunosuppression, multiple immunotherapy mechanisms, PD-1 axis driven, highly inflamed, and immune desert based upon PD-L1 IHC results and manual review of expression profiles.

\section{Results}

\section{Immune Infiltrate}

The immune infiltrate encompasses a variety of cell types, including CD8+ CTLs, CD4+ Tregs, type 1 and 2 macrophages, neutrophils and others. In this study RNA-seq was used to quantify cell types in the tumor microenvironment, while IHC was used to identify spatial characteristics of CD8+ T-cells. The gene expression of tumor-infiltrating $\mathrm{CD}^{+}$CTLs in PDAC as compared to patient tumors from a reference population was not remarkably different $(\mathrm{P}=0.5855)$. More than onethird of all PDAC $(24 / 68 ; 35.3 \%)$ were characterized by CD8 ${ }^{+}$T-cell counts supporting a lack of effector T-cells (rank <25; https://cdn.amegroups.cn/static/application/ 7781b443d858980153cd7fd01cb26552/ATM-20-1076-
Supplementary2.pdf), while a minority were considered as having a high number of such cells (10/68, 14.7\%; rank 275$)$. By comparison $\mathrm{CD}^{+}{ }^{+} \mathrm{T}$-cells were high in more than one-third of all PDAC $(27 / 68 ; 35.7 \%)$, with only a minority considered as having a low number of such cells $(8 / 68,11.8 \%$; rank 25). For type 2 macrophage (M2) content (CD163) slightly more than one-fourth of all PDAC $(19 / 68 ; 27.9 \%)$ showed a high to very high expression (rank 275 ). For 10 PDAC without a significant number of M2 macrophages (rank <25), 6 also had a very low number of $\mathrm{CD}^{+}$and $\mathrm{CD}^{+}{ }^{+} \mathrm{T}$-cells and could be best considered as an "immune desert" (26). Markers of neutrophilic differentiation, activation, or recruitment including SRC kinase associated phosphoprotein 2 (SKAP2; $\mathrm{P}=3.47544 \mathrm{E}-14)$, leucine rich alpha-2-glycoprotein 1 (LRG1; $\mathrm{P}=0.0166)$, or kringle containing transmembrane protein 1 (KREMEN1; $\mathrm{P}=0.0300)$ had mean expression ranks higher in the PDAC as compared to the reference population, including the aforementioned immune desert cases (https://cdn.amegroups.cn/static/application/77 81b443d858980153cd7fd01cb26552/ATM-20-1076Supplementary2.pdf), potentially pointing to neutrophils as an additional source of myeloid suppression in this context. The overall immune infiltrate results support a tumor microenvironment commonly deficient in $\mathrm{CD} 8^{+} \mathrm{T}$-cells but not $\mathrm{CD}^{+}{ }^{+} \mathrm{T}$-cells and frequently dominated by a myeloid population of Type 2 macrophage immunosuppressive cells.

The pattern of $\mathrm{CD}^{+}{ }^{+} \mathrm{T}$-cell infiltration by IHC, however showed an equal mix of infiltrating $(34 / 68 ; 50 \%)$ and noninfiltrating cases (Figure 1; https://cdn.amegroups.cn/static/ application/7781b443d858980153cd7fd01cb26552/ATM20-1076-Supplementary2.pdf). The excluded pattern of infiltration whereby T-cells were limited to the tumor stroma interface was not identified in any PDAC case, although it was seen in the comparison cohort.

\section{CTLA-4 immune checkpoint}

T-cell activation is a complex process that balances multiple stimulatory and inhibitory signals that begins with the T-cell receptor (TCR) on T-cells binding to a major histocompatibility complex (MHC) on antigen presenting cells (APC) that provides specificity to the process. CTLA-4, a T-cell co-inhibitory receptor, competes with CD28, a T-cell co-stimulatory receptor, for the binding of B7-1 (CD80) or B7-2 (CD86) ligands on the APC. CTLA-4 has a higher binding affinity than CD28 for these ligands and the balance of stimulatory and inhibitory signals results 

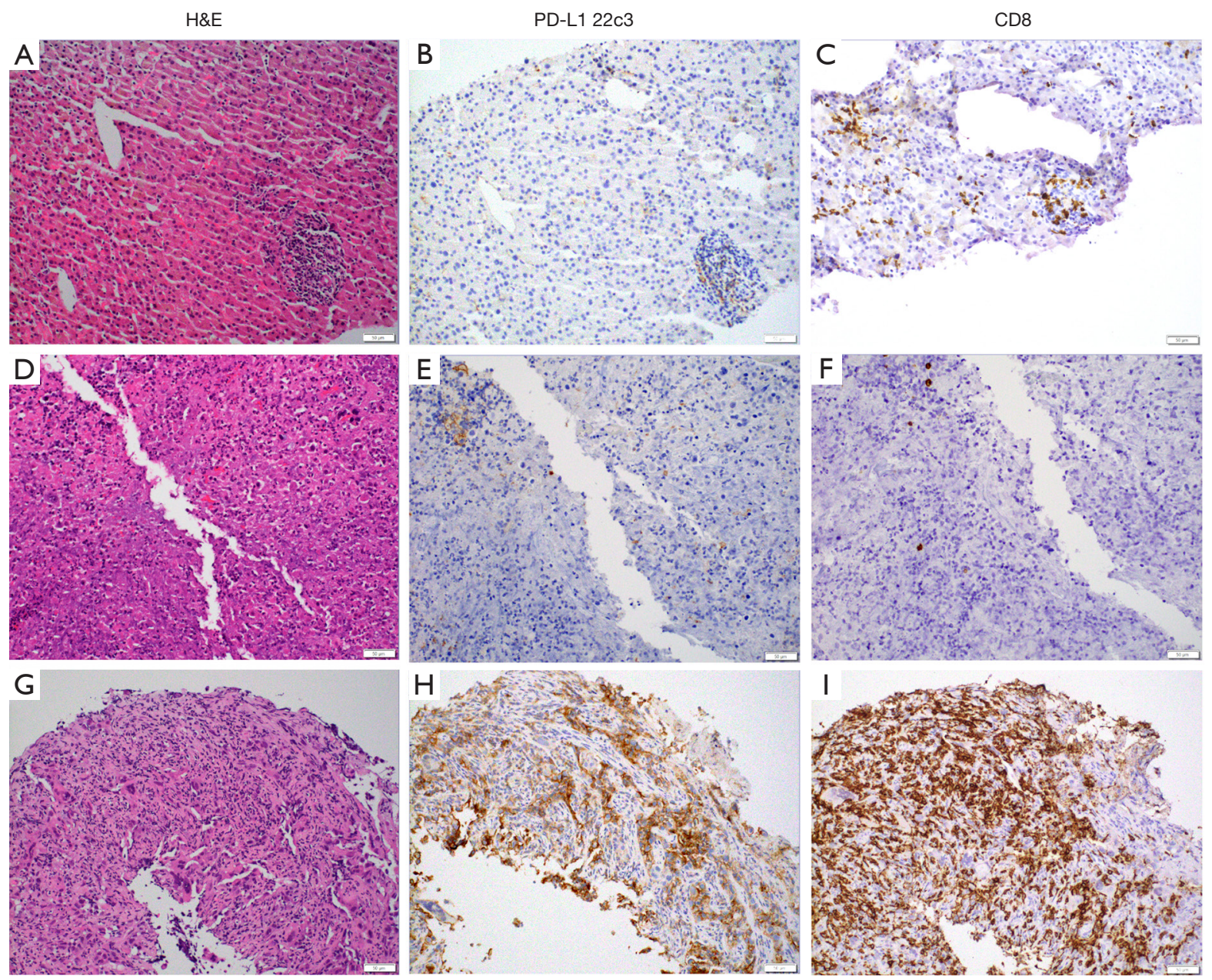

Figure 1 Tumor-infiltrating CD8+ CTLs in PDAC. Immunohistochemistry for CD8 and H/E-stained sections show a highly inflamed and a non-inflamed case. Scale bar $=100 \mu \mathrm{m}$. PDAC, pancreatic ductal adenocarcinoma.

in the net signal of suppression versus T-cell activation, respectively. The majority $(61.8 \% ; 42 / 68)$ of the PDAC samples evaluated for CTLA-4 and CD28 expression by RNA-seq showed a higher level of the latter receptor indicating an overall $\mathrm{T}$-cell activation process through this pathway. Additionally, CTLA-4 was at maximal levels of moderately high in only 12 of 68 (17.6\%) of PDAC samples, for which all but three showed very similar levels of CD28. The overall results support that CTLA-4 immune checkpoint is an immunosuppressive mechanism in PDAC, but in a minority of cases.

\section{PD-1 Axis immune checkpoint}

PD-1, and its ligands PD-L1 and PD-L2 members of the B7/CD28 family of receptors deliver a co-inhibitory signal to effector T-cells. Unlike CTLA-4, the expression of PD-1 and ligands is not limited to T-cells and APC, but are also expressed on B-cells, myeloid cells, and neoplastic cells. The majority $(69.1 \% ; 47 / 68)$ of PDAC samples evaluated for PD-L1 expression by IHC had no staining in neoplastic cells, with the remaining samples having staining in $1 \%$ or more of neoplastic cells. Among PD-L1 IHC positive cases, four showed a strong PD-L1 IHC result, i.e., greater than $50 \%$ staining in neoplastic cells. These IHC results were confirmed by RNA-seq analysis for PD-L1 showing a less than high level of expression for the majority (44/47; 93.6\%) of IHC negative cases and a high expression for the majority $(9 / 14 ; 64.3 \%)$ of cases with $5 \%$ or more of neoplastic cells staining. The discordant results between IHC and RNA-seq for these other five cases is not readily explained other than perhaps a lack of sensitivity of the 
22C3 clone for PDAC (27). These results suggest that PD-1 axis checkpoint blockade is a factor in a subset of PDAC, and while staining in $1 \%$ or more of neoplastic cells can be considered a positive result there are undoubtedly an estimated $5 \%$ or so of cases with very high PD-L1 expression.

\section{Alternative immune checkpoint}

In addition to PD-1 and CTLA-4 immune checkpoint receptors, there are additional inhibitory receptors (LAG3, TIM3, VISTA) preferentially expressed on either T- cells (LAG3, TIM3) or myeloid cells (VISTA). Animal models have shown enhanced antitumor immune responses after blockade of these additional inhibitory receptors when combined with blockade of PD-1 or CTLA-4 and more recently human clinical trial reports for LAG3 inhibition in melanoma have shown encouraging results (28-30). The prevalence of moderately high or high expression for either TIM3 or VISTA was 29.4\% (20/68) and 63.2\% (43/68), respectively, while LAG3 was only highly expressed in six cases $(6 / 68 ; 8.8 \%)$ (Table 1$)$. The lack of expression of LAG3 and its nearly exclusive expression on T-cells is consistent with the prior similar results for CTLA-4. This compares to the expression pattern of VISTA on myeloid cells, as well as pancreatic ducts, implying the significant role that myeloid suppression has in PDAC (31). The overall results support alternative immune checkpoint as having a role in PDAC, but primarily via TIM3 or VISTA and not LAG3.

\section{Immune checkpoint activation}

CTL-dependent tumor eradication requires the TCRdependent recognition of a tumor-associated antigen (TAA) or neoantigen presented by dendritic cells (DCs) or other antigen-presenting cells (APCs) in the context of MHC Class I molecules, along with activation signals via one or multiple co-stimulatory ligand and receptor pairs expressed by CTLs and associated APCs, respectively, including CD137 ligand (CD137L) and CD137, TNFSF7 (CD27 Ligand/CD27L; also known as CD70) and CD27, TNFSF18 (GITR Ligand/GITRL) and GITR, CD40 ligand (CD40L) and CD40, OX40 ligand (OX40L) and OX40, and inducible costimulator-ligand (ICOSL) and ICOS. Activation of specific co-stimulatory receptors, including CD27, CD137, OX40 and GITR have been shown to promote the differentiation of $\mathrm{CD}^{+}{ }^{+} \mathrm{TH} 1$ or TH9 $\mathrm{T}$ cells with anticancer activity while suppressing the differentiation or function of $\mathrm{CD}^{+} \mathrm{CD} 25^{+} \mathrm{FOXP} 3^{+}$TREG cells and subsequent myeloid suppression (32-35).

In this series of PDAC the prevalence of over-expression of the immune checkpoint activation receptors was somewhat common (CD137 16.2\%, CD27 20.6\%, CD40 $30.9 \%$, ICOS $22.1 \%$; Table 1) and at least one of these genes was highly or moderately highly expressed in more than half of all cases $(36 / 68 ; 52.9 \%)$. While over-expression of at least one co-stimulatory T-cell signal was quite common this finding was not remarkably different from the comparison cohort (Table 1), with the exception that GITR had significantly lower expression in PDAC $(\mathrm{P}=0.0283)$. As will be shown later in categories of immunosuppression in PDAC, the over expression of co-stimulatory T-cell signals is common in some groups, but uncommon in a unique group with combined metabolic and myeloid immunosuppression. To summarize, immune checkpoint activation by specific co-stimulatory receptors, including CD27, CD137, OX40, CD40, and ICOS, but not GITR, is a frequent event in certain subgroups of PDAC.

\section{Myeloid immunosuppression}

While there are many types of myeloid cells in the tumor microenvironment, most notable are TAMs, which consist of inflammatory (M1) and immunosuppressive (M2) phenotypes. These two types of TAMs have distinct patterns of cytokine expression with M1 TAMs noted for secretion of interferon gamma (IFN $\gamma$ ), IL-6, and chemokine (C-X-C motif) ligand 10 (CXCL10). While M2 TAMs secrete transforming growth factor beta 1 (TGFB1; best known as TGF- $\beta 1$ ), interleukin 10 (IL10), chemokine (CC motif) ligand 22 (CCL22), and promote indoleamine 2,3-dioxygenase 1 (IDO1) production (36). Additionally, colony stimulating factor 1 receptor (CSF1R), CCR2, and CCL2 produced by M2 macrophages and neoplastic cells, promote the recruitment of additional monocytes to the tumor microenvironment, their proliferation, and differentiation towards the M2 immunosuppressive phenotype, in a self-propagating process (37).

In this series of PDAC, the mean rank expression value of 61.4 for M2 markers (TGBF1, IL10, CCL22, IDO1, CSF1R, CCR2, CCL2) was nearly double the value of 38.3 for M1 markers (IFN gamma, IL-6, CXCL10) supporting the significant role that myeloid suppression has in PDAC. Among this list of myeloid immunosuppressive markers, several are the specific target of immunomodulatory immunotherapeutics including IL-10, TGFB1, CSF1R, 
and CCR2/CCL2. Among this list, CRR2 and its ligand CCL2, CSF1R, and TGFB1 were moderately-high to highly expressed in more than one-half of all cases, with the latter (TGFB1) being the most frequently over expressed in comparison to the reference population. Using a Wilcoxon rank-sum test to compare the PDAC patient cohort with the reference population for the expression rank of all genes evaluated, multiple $\mathrm{M} 2$ markers were expressed significantly higher in PDAC samples as compared to the remaining tumor types tested including CCR2 $(\mathrm{P}=0.0316)$, CCL2 ( $\mathrm{P}=4.16123 \mathrm{E}-05)$, and TGFB1 ( $\mathrm{P}=0.0001)$ (Table 1). As a group at least one gene from this list of M2 markers was moderately-high to highly expressed for all cases. Expression values for macrophage content (CD68, CD163) paralleled this over expression of myeloid suppressive cytokines and chemokines being higher than CD8 values for all but nine of sixty-four cases. Overall, myeloid immunosuppression is a dominant theme in PDAC and will be an important target in future immunomodulatory immunotherapeutic development in this tumor type.

\section{Metabolic immunosuppression}

Cell death releases ATP into the tumor microenvironment that is rapidly degraded to adenosine by the canonical adenosinergic pathway of ectonucleotidases CD39 and CD73. CD39 is preferentially expressed on regulatory $\mathrm{T}$ cells and activated NKT cells and macrophages, while CD73, is highly expressed by neoplastic cells, endothelial cells, regulatory $\mathrm{T}$ cells, and some B cells. A less well investigated non-canonical adenosinergic pathway exists whereby cyclic ADP-ribose hydrolase 1 (CD38) converts $\mathrm{NAD}^{+}$to ADPR, which is then converted by CD203a (PC1) to AMP, that is subsequently metabolized to adenosine by CD73, with the latter representing the common link between these two pathways. The non-canonical adenosinergic pathway has typically been characterized as functional in hematopoietic neoplasms, most notably myeloma, but has also been shown to be functional in melanoma (38). The final substrate of both pathways, adenosine, directly inhibits the activation of macrophages by inflammatory stimuli and promotes remodeling to an M2-like phenotype via multiple adenosine receptors, most notably ADORA2A (39).

In this series of PDAC, most cases $(61 / 68 ; 89.7 \%)$ showed high or moderately-high expression of CD73, with similar expression of CD39 (32/68; 47.1\%). Among this list of metabolic immunosuppressive markers ADORA2A and CD38 are targets of immunomodulatory immunotherapeutics. However, ADORA2A and CD38 were infrequently over-expressed $(9 / 68 ; 13.2 \%$ and $7 / 68$; $10.3 \%$, respectively) relegating much of the potential clinical efficacy of targeting this immunosuppressive pathway to the upstream ectonucleotidases CD39 and CD73. The uniqueness of these two therapeutic targets is shown by the significant difference of $\mathrm{CD} 39(\mathrm{P}=0.0001)$ and CD73 $(\mathrm{P}=1.0509 \mathrm{E}-06)$ expression as compared to the reference population (Table 1) supports that the canonical adenosinergic pathway is more commonly used in PDAC.

\section{$T M B$, microsatellite instability, and PD-L1/2 copy number}

TMB was available for 64 of 68 cases with four cases having a low neoplastic cell content $(<20 \%)$ that precludes definitive evaluation. Defining high mutational burden as the upper tenth percentile resulted in seven PDAC classified as high TMB (https://cdn.amegroups.cn/static/ application/7781b443d858980153cd7fd01cb26552/ATM20-1076-Supplementary2.pdf) $(40,41)$. For cases with high TMB there was no association with a specific type of immunosuppression and all were MSS with one exception where results were not available. Results for microsatellite instability were available for fifty-eight patients and for which all were stable. Results for $P D-L 1 / 2$ amplification were available for fifty-four patients and none were amplified.

\section{Clinically relevant immunosuppression expression profiles}

While each case represented a unique repertoire of molecular and immune biomarkers there were frequent similarities of overall results that could be summarized into at least five categories of immunosuppression (Figure 2).

\section{Myeloid and metabolic immunosuppression}

As the most common subgroup $(25 / 68 ; 36.8 \%)$, cases in this group show immunosuppressive mechanisms limited to both myeloid and metabolic immunosuppression (Figure 2). There was minimal to no evidence of PD-1 axis expression, alternative checkpoint blockade, or co-stimulatory T-cell signaling, and cases were typically exemplified by over expression of both TGFB1 and CD73. These cases typically had a paucity of $\mathrm{CD} 8^{+} \mathrm{T}$-cells while having a high number of $\mathrm{CD}^{+}$T-cells and high macrophage content. Notably 


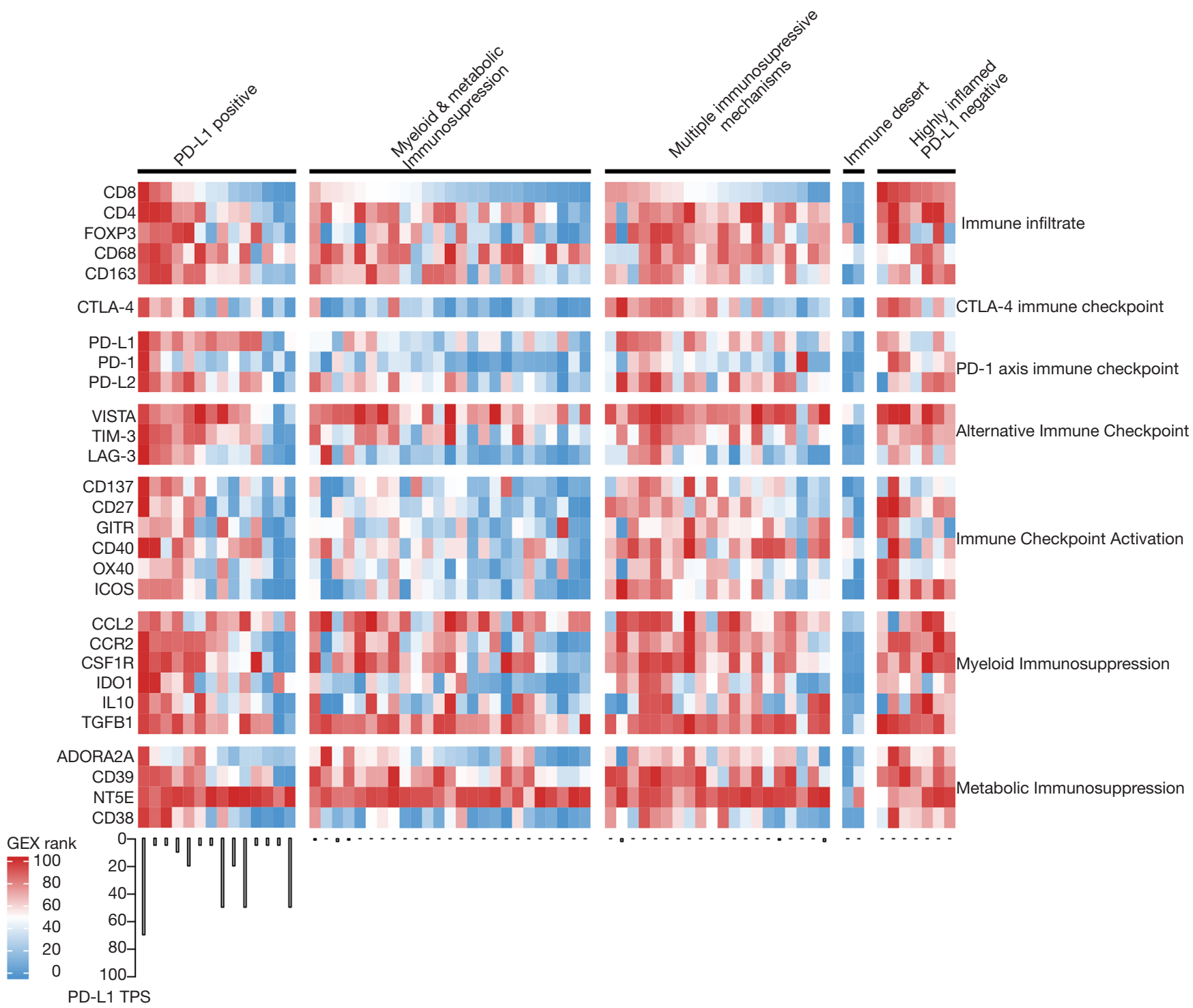

Figure 2 Clinically relevant immune gene expression profiles in PDAC. Expression rank of 23 genes in 68 PDAC patients as compared to a comparison cohort expression database of multiple tumor types $(n=1,416)$. Seven different classes of actionable genes including immune infiltrate, CTLA-4 immune checkpoint, PD-1 axis immune checkpoint, alternative immune checkpoint, immune checkpoint activation, myeloid immunosuppression, and metabolic immunosuppression were used to classify cases into a clinically relevant framework. The clinically relevant immunosuppression expression profiles were combined myeloid metabolic immunosuppression and multiple immunotherapy mechanisms representing slightly more than two-thirds of all cases, followed by PD-1 axis driven, highly inflamed and immune desert. PDAC, pancreatic ductal adenocarcinoma. 
absent from this group was over expression of the costimulatory T-cell signals CD27, CD137, OX40, CD40, ICOS, and GITR.

\section{Multiple immunosuppressive mechanisms}

The second most common group $(20 / 68 ; 29.4 \%)$, cases with multiple mechanisms of immunosuppression typically showed a modest number of $\mathrm{CD} 8^{+} \mathrm{T}$-cells, a relatively much higher number of $\mathrm{CD}^{+} \mathrm{FOXP}^{+}{ }^{+} \mathrm{T}$-cells, high macrophage content, alternative checkpoint blockade, co-stimulatory T-cell signaling, a dominant myeloid suppression coupled with metabolic adenosinergic immunosuppression, and less than significant PD-1 axis checkpoint blockade. These cases are best described as having multiple mechanisms of immunosuppression (Figure 2) and while this supports more than one immunotherapy approach, the optimal sequential order of combination immunotherapies for this group is somewhat difficult to interpret.

\section{PD-L1 positive}

PD-L1 positive, representing a minority of cases (14/68; $20.6 \%$ ) showed $5 \%$ or greater expression of PD-L1 by IHC in neoplastic cells. The number of CD8 and CD4 T-cells for each case was highly variable in this group, with low to moderately low numbers of both of these important subsets of TILs in a substantial number of cases (4/14) representing what could be referred to as non-inflamed, or cold, PD-L1 positive PDAC. As expected, expression of other immunotherapeutic targets in this group more closely aligned with the number of TILs and not with the level of PD-L1 IHC expression.

\section{Highly inflamed PD-L1 negative}

This group, characterized by an abundance of both CD8 and CD 4 T-cells and negative PD-L1 by IHC, were uncommon $(7 / 68 ; 10.3 \%)$, but still represented a substantial number of all cases (Figure 2). Similar to the cases with multiple mechanisms of immunosuppression there was over expression of multiple immunotherapeutic targets. Overall these cases represented a highly inflamed tumor microenvironment that is unusual for PDAC and often seen in more immunogenic tumor types such as melanoma or lung cancer.

\section{Immune desert}

There were two cases that met the definition of a low number of both CD8 and CD4 T-cells with low or moderately-low expression of not more than one immunotherapeutic target. One sample showed moderately high or greater expression of CD73 and GITR (Figure 2).

\section{Conclusions}

Immune checkpoint inhibitors targeting CTLA4, PD1, and PD-L1 employed as standalone interventions have currently provided limited clinical benefit to subjects with PDAC $(1,2,42)$. Three phase 1 or 2 trials reported the investigational use of either ipilimumab, pembrolizumab, or the investigational PD-L1 inhibitor BMS-936559 with total enrollment of less than 60 PDAC patients $(1,2,42)$. No responders were identified after treatment with ipilimumab or the investigational PD-L1 inhibitor BMS936559, although three partial responders from seventeen patients were identified in the pembrolizumab study and for which PD-L1 expression was not reported. This should not be interpreted to mean that single agent $\mathrm{PD}-1$ axis intervention has no role in future PDAC immunotherapy but, rather, that identifying specific patients with a PD-L1driven phenotype may be necessary to derive benefit. In our case series, one-fifth of PDAC patients showed evidence of PD-1 axis-driven immunosuppression by $5 \%$ or greater expression of PD-L1 by IHC in neoplastic cells that could be favorable for response to targeting PD-L1 or PD-1. The RNA-seq analysis for these cases showed that greater than one-fourth of these $\mathrm{PD}-\mathrm{L} 1$ positive PDAC were either noninflamed or at most weakly inflamed. At the current time the impact of number of TILs in PD-L1 positive PDAC to response to ICIs is unknown, but is likely important. Nonetheless, our series of 68 PDAC shows approximately 10 to $15 \%$ of cases are inflamed PD-L1 positive and should be considered as a unique group for future trial enrollment.

Our study supports immunotherapeutic interventions that target myeloid and metabolic immunosuppression should lead to clinical benefit for a proportion of PDAC patients. There are (at the time of writing) more than twenty clinical trials targeting the myeloid-associated targets of CCL2/CCR2, CSF1R, TGFB1, IDO1, or $\mathrm{TNF}$, and are ongoing and/or enrolling patients with pancreatic cancer (Table 2; source https://clinicaltrials. gov). Prior results in PDAC point to the CCL2/CCR2 axis as a promising actionable pathway in this setting. Results from at least one single institution Phase I clinical trial focused on CCR2 blockade in this oncological indication is available (43). In this study, the CCR2 inhibitor PF04136309 was given in combination with FOLFIRINOX, achieving $42 \%$ objective response rate $(n=33)$ as 
Table 2 Examples of immunotherapeutic targets beyond PD-L1 and associated interventions and clinical trials with eligibility of advanced solid tumors or PDAC

\begin{tabular}{|c|c|c|c|c|}
\hline Function & Target & Drug & $\begin{array}{l}\text { Mechanism of } \\
\text { action }\end{array}$ & Clinical trial \\
\hline \multirow[t]{3}{*}{$\begin{array}{l}\text { Alternative immune } \\
\text { checkpoint }\end{array}$} & LAG3 & $\begin{array}{l}\text { Relatlimab; BI 754111; BMS-986213; } \\
\text { FS118; INCAGN02385; LAG525; } \\
\text { MGD013; MK-4280; REGN3767; } \\
\text { Sym022; TSR-033 }\end{array}$ & Antagonist & $\begin{array}{l}\text { NCT02460224, NCT02720068, } \\
\text { NCT03005782, NCT03156114, } \\
\text { NCT03219268, NCT03250832, } \\
\text { NCT03335540, NCT03440437, } \\
\text { NCT03459222, NCT03489369, } \\
\text { NCT03538028, NCT03607890 }\end{array}$ \\
\hline & TIM3 & $\begin{array}{l}\text { BMS-986258; LY3321367; MBG453; } \\
\text { Sym023; TSR-022 }\end{array}$ & Antagonist & $\begin{array}{l}\text { NCT02608268, NCT02791334, } \\
\text { NCT02791334, NCT02817633, } \\
\text { NCT03099109, NCT03446040, } \\
\text { NCT03489343, NCT03652077 }\end{array}$ \\
\hline & VISTA & CA-170 & Antagonist & NCT02812875 \\
\hline \multirow{2}{*}{ Checkpoint activation } & CD40 & $\begin{array}{l}\text { Selicrelumab; ABBV-428; ABBV-927; } \\
\text { ADC-1013; APX005M; CDX-1140; } \\
\text { MEDI5083; SEA-CD40 }\end{array}$ & Agonist & $\begin{array}{l}\text { NCT02304393, NCT02376699, } \\
\text { NCT02588443, NCT02665416, } \\
\text { NCT02829099, NCT02955251, } \\
\text { NCT02988960, NCT03089645, } \\
\text { NCT03193190, NCT03214250, } \\
\text { NCT03329950, NCT03502330 }\end{array}$ \\
\hline & GITR & $\begin{array}{l}\text { BMS-986156; GWN 323; } \\
\text { INCAGN01876; MK-4166; OMP- } \\
\text { 336B11; TRX518 }\end{array}$ & Agonist & $\begin{array}{l}\text { NCT01239134, NCT02628574, } \\
\text { NCT02697591, NCT02740270, } \\
\text { NCT03126110, NCT03295942, } \\
\text { NCT03335540 }\end{array}$ \\
\hline \multirow{4}{*}{ Myeloid suppression } & IDO1 & $\begin{array}{l}\text { Epacadostat; Indoximod; BMS- } \\
\text { 986205; KHK2455; LY3381916; MK- } \\
\text { 7162; NLG802 }\end{array}$ & Antagonist & $\begin{array}{l}\text { NCT02658890, NCT02867007, } \\
\text { NCT03164603, NCT03217669, } \\
\text { NCT03322384, NCT03335540, } \\
\text { NCT03343613, NCT03364049, } \\
\text { NCT03459222, NCT03589651 }\end{array}$ \\
\hline & TGFB1 & $\begin{array}{l}\text { Galunisertib; M7824; NIS793; SAR- } \\
439459\end{array}$ & Antagonist & $\begin{array}{l}\text { NCT02423343, NCT02517398, } \\
\text { NCT02947165, NCT03192345, } \\
\text { NCT03436563, NCT02734160 }\end{array}$ \\
\hline & TNF & $\begin{array}{l}\text { Certolizumab; Lenalidomide; } \\
\text { Thalidomide }\end{array}$ & Antagonist & NCT01661400 \\
\hline & CSF1R & $\begin{array}{l}\text { Cabiralizumab; Emactuzumab; } \\
\text { AMG 820; ARRY-382; BLZ945; JNJ- } \\
\text { 40346527; LY3022855; PD-0360324; } \\
\text { SNDX-6352 }\end{array}$ & Antagonist & $\begin{array}{l}\text { NCT02554812, NCT02829723, } \\
\text { NCT03193190, NCT03238027, } \\
\text { NCT03335540, NCT03336216, } \\
\text { NCT03431948, NCT03502330, } \\
\text { NCT03599362 }\end{array}$ \\
\hline
\end{tabular}

Table 2 (continued) 
Table 2 (continued)

\begin{tabular}{lllll}
\hline Function & Target & Drug & $\begin{array}{l}\text { Mechanism of } \\
\text { action }\end{array}$ & Clinical trial \\
\hline Metabolic suppression & ADORA2A & AZD4635; CPI-444; NIR178; PBF- & Antagonist & NCT02740985, NCT03207867, \\
& CD39/CD73 & $\begin{array}{l}\text { 509; MEDI9447; BMS-986179; CPI- } \\
\text { NC6 }\end{array}$ & & $\begin{array}{l}\text { NCT02503754451, NCT03334617, } \\
\text { NCT03381274, NCT03611556 }\end{array}$ \\
& & & & NCT02799095 \\
Immune deserts & CD8 & Etirinotecan Pegol; ALKS 4230; ALT- & Immunostimulator & \\
& & 803; NKTR-214 & &
\end{tabular}

PDAC, pancreatic ductal adenocarcinoma.

compared to no objective responses in patients receiving FOLFIRINOX alone $(\mathrm{n}=5)$ (NCT01413022), and to the historic response rate of FOLFIRINOX alone (31\%) (44). In the context of this novel treatment regimen, PF-04136309 efficiently disrupted CCL2/CCR2 signaling and inhibited the intra-tumoral accumulation of immunosuppressive CCR2 ${ }^{+}$TAMs, which was paralleled by an increase in $\mathrm{CD} 8^{+}$ T-cell infiltration (recapitulating preclinical data with PF04136309) $(15,16,45)$. Importantly, in our study CCR2 and CCL2 were significantly over expressed in PDAC compared to the comparison cohort.

In a comparable fashion, the TGFB 1 inhibitor galunisertib has shown a survival advantage combined with gemcitabine as front line therapy. In a recently reported phase $1 \mathrm{~b} / 2$ study patients were randomised $2: 1$ to galunisertib-gemcitabine $(n=104)$ or placebogemcitabine $(\mathrm{n}=52)$ with median survival times of 8.9 and 7.1 months for galunisertib and placebo, respectively (46). More importantly, when galunisertib-gemcitabine treated patients were evaluated for TGFB1 response there was a greater difference in survival of 10.1 months for responders and 6.7 months for non-responders. To summarize, previously completed trials in PDAC and our current study would support that targeting myeloid and metabolic immunosuppression should lead to clinical benefit for a proportion of subjects with PDAC.

Our findings also point to CD39 and CD73, which are overexpressed by $\mathrm{T}_{\mathrm{REG}}$ and malignant cells, respectively, as potential actionable targets. CD39 and CD73 are ectonucleotidases that through the conversion of ADP/ ATP to AMP and AMP to adenosine, respectively, result in a shift from an ATP-driven pro-inflammatory environment to an anti-inflammatory state. Adenosine suppresses antitumor immunity by binding to adenosine receptors (A1, A2a, A2b, A3), among which A2aR, or ADORA2A, is the predominant cell surface immune checkpoint expressed on the surface of T-cells (47). A recent study using an azido-labeled bioorthogonal chemical reporter to metabolically label $\mathrm{N}$-linked glycoproteins on the surface of pancreatic cancer cell lines to identify potential tumor-specific targets identified CD73 as a leading candidate (48). Additionally, there are more than twenty clinical trials targeting alternative checkpoint blockade (TIM3, LAG3, VISTA) with eligibility of advanced solid tumors applicable to PDAC, with one prior study supporting that within this group of targets that VISTA is over expressed in comparison to a more immunogenic (melanoma) tumor type (melanoma) (49). Our results support this conclusion with 43 of 68 PDAC patients $(63 \%)$ demonstrating overexpression of VISTA, and with much lower rates for TIM3 or LAG3. What was not shown in this prior study, but identified in our results is that over expression of VISTA is rarely if ever a singular event and typically seen in cases with a predominant myeloid and metabolic or multiple mechanisms of immunosuppression. In these scenarios, the use of VISTA antagonists will likely be complicated by other immunosuppressive mechanisms, although there were instances where this target was over expressed in PD-1 axis driven tumors and the dual PD-1/VISTA inhibitor CA-170 could be more uniquely considered.

This study with 68 patients was comparable in size to some prior studies of mRNA expression profiling in PDAC, but considerably smaller than prior studies performed by The Cancer Genome Atlas Project. Our study in comparison to these prior studies is unique in its focus on immunotherapeutics that are currently FDAapproved or in human clinical trials. Prior classification schemas such as the four-group classification of squamous, immunogenic, pancreatic progenitor, or aberrantly differentiated exocrine of Bailey et al., the three-group classification (classical, quasimesenchymal, or exocrine-like) of Collisson et al., and the two-group classification of basal- 
like or classical of Moffitt et al. (50), do not readily lend themselves to clinical applications $(40,51)$. Gene targets will evolve as new immunotherapeutic agents enter into human clinical trials, but the overall approach of a clinically directed comprehensive immune profiling will remain the same. Nonetheless, the overall findings of this study are consistent with the available literature, which reports the overexpression of ectonucleotidases, such as CD39 and CD73, as well as the presence of various biomarkers of myeloid suppression, i.e., CSF1R, and a prominent macrophage component, in PDAC (11). It should also be noted that while uncommon, there are examples of inflamed PD-1 axis checkpoint blockade-driven PDAC. These specific cases, which can only be properly classified by a comprehensive approach as performed in this study, are likely similar to more immunogenic tumor types like melanoma and lung cancer. This would imply a subset of PDAC do exist that are potentially responsive to checkpoint inhibitors and could readily be enrolled in PD-1 axis checkpoint blockade clinical trials.

To summarize, the strength of this study is demonstration that a unique profile of immune markers can be identified in PDAC and used to direct personalized immunotherapeutic decision-making strategies. Themes that emerge in the current study in patients with PDAC include myeloid and metabolic suppression, and expression of a variety of checkpoints. This study demonstrates that a unique profile of immune markers can be identified in PDAC and used to direct personalized immunotherapeutic decision-making strategies. While some types of immune profiles may be more or less common, ultimately, optimal therapeutic targeting should be based on the immune profile of the individual patient to be treated, a concept that is central to the tenets of personalized cancer therapy.

\section{Acknowledgments}

Funding: The research was funded by OmniSeq, Inc. (Buffalo, NY).

\section{Footnote}

Reporting Checklist: The authors have completed the MDAR reporting checklist. Available at http://dx.doi.org/10.21037/ atm-20-1076

Data Sharing Statement: Available at http://dx.doi. org/10.21037/atm-20-1076
Conflicts of Interest: All authors have completed the ICMJE uniform disclosure form (available at http://dx.doi. org/10.21037/atm-20-1076). FLL, SP, PDP, MN, JC, BB, $\mathrm{SG}$, and $\mathrm{CM}$ are all employees of OmniSeq, Inc. (Buffalo, $\mathrm{NY}$ ) and hold restricted stock in OmniSeq, Inc. JC, SG, BK, and $\mathrm{CM}$ are employees of Roswell Park Comprehensive Cancer Center (Buffalo, NY). Roswell Park Comprehensive Cancer Center is the majority shareholder of OmniSeq, Inc. RK receives research funding from Incyte, Genentech, Merck, Serono, Pfizer, Sequenom, Foundation Medicine, and Guardant, as well as consultant fees from X Biotech, Loxo, NeoMed, and Actuate Therapeutics, speaker fees from Roche, and has an ownership interest in Curematch Inc. Dr. Nesline reports other from OmniSeq, Inc., outside the submitted work. Dr. Kato reports other from null, during the conduct of the study. Dr. Pabla reports other from Omniseq, outside the submitted work; In addition, Dr. Pabla has a patent 20200048717 pending, and a patent 20180107786 pending. Dr. Kurzrock reports other from Dr. Kurzrock has the following disclosure information: Stock and Other Equity Interests. (IDbyDNA, CureMatch, Inc., and Soluventis); Consulting or Advisory Role (Gaido, LOXO, X-Biotech, Actuate Therapeutics, Roche, NeoMed, Soluventis, Pfizer, and Merck); Speaker's fee (Roche); Research Funding (Incyte, Genentech, Merck Serono, Pfizer, Sequenom, Foundation Medicine, Guardant Health, Grifols, Konica Minolta, DeBiopharm, Boerhringer Ingelheim, and OmniSeq [All institutional]); Board Member (CureMatch, Inc., and CureMetrix, Inc.)., during the conduct of the study. The other authors have no conflicts of interest to declare.

Ethical Statement: The authors are accountable for all aspects of the work in ensuring that questions related to the accuracy or integrity of any part of the work are appropriately investigated and resolved. OmniSeq's analysis utilized deidentified data that was considered non-human subjects research in accordance with institutional local IRB requirements (IRB-approved protocol BDR \#073166) and the Helsinki declaration (as revised in 2013) at Roswell Park Comprehensive Cancer Center (Buffalo, NY).

Open Access Statement: This is an Open Access article distributed in accordance with the Creative Commons Attribution-NonCommercial-NoDerivs 4.0 International License (CC BY-NC-ND 4.0), which permits the noncommercial replication and distribution of the article with the strict proviso that no changes or edits are made and the original work is properly cited (including links to both the 
formal publication through the relevant DOI and the license). See: https://creativecommons.org/licenses/by-nc-nd/4.0/.

\section{References}

1. Royal RE, Levy C, Turner K, et al. Phase 2 Trial of Single Agent Ipilimumab (Anti-CTLA-4) for Locally Advanced or Metastatic Pancreatic Adenocarcinoma. J Immunother 2010;33:828-33.

2. Brahmer JR, Tykodi SS, Chow LQM, et al. Safety and Activity of Anti-PD-L1 Antibody in Patients with Advanced Cancer. N Engl J Med 2012;366:2455-65.

3. Patel SP, Kurzrock R. PD-L1 Expression as a Predictive Biomarker in Cancer Immunotherapy. Mol Cancer Ther 2015;14:847-56.

4. Vonderheide RH, Bayne LJ. Inflammatory networks and immune surveillance of pancreatic carcinoma. Curr Opin Immunol 2013;25:200-5.

5. Clark CE, Beatty GL, Vonderheide RH. Immunosurveillance of pancreatic adenocarcinoma: Insights from genetically engineered mouse models of cancer. Cancer Lett 2009;279:1-7.

6. Dougan SK. The Pancreatic Cancer Microenvironment. Cancer J 2017;23:321-5.

7. Galluzzi L, Zitvogel L, Kroemer G. Immunological Mechanisms Underneath the Efficacy of Cancer Therapy. Cancer Immunol Res 2016;4:895-902.

8. Bantug GR, Galluzzi L, Kroemer G, et al. The spectrum of $\mathrm{T}$ cell metabolism in health and disease. Nat Rev Immunol 2018;18:19-34.

9. Galluzzi L, Baehrecke EH, Ballabio A, et al. Molecular definitions of autophagy and related processes. EMBO J 2017;36:1811-36.

10. Buqué A, Bloy N, Aranda F, et al. Trial Watch: Immunomodulatory monoclonal antibodies for oncological indications. Oncoimmunology 2015;4:e1008814.

11. Künzli BM, Berberat PO, Giese T, et al. Upregulation of CD39/NTPDases and P2 receptors in human pancreatic disease. Am J Physiol Gastrointest Liver Physiol 2007;292:G223-30.

12. Vacchelli E, Bloy N, Aranda F, et al. Trial Watch: Immunotherapy plus radiation therapy for oncological indications. Oncoimmunology 2016;5:e1214790.

13. Feig C, Jones JO, Kraman M, et al. Targeting CXCL12 from FAP-expressing carcinoma-associated fibroblasts synergizes with anti-PD-L1 immunotherapy in pancreatic cancer. Proc Natl Acad Sci 2013;110:20212-7.

14. Garg AD, More S, Rufo N, et al. Trial watch:
Immunogenic cell death induction by anticancer chemotherapeutics. Oncoimmunology 2017;6:e1386829.

15. Nywening TMM, Wang-Gillam A, Sanford DEE, et al. Targeting tumour-associated macrophages with CCR2 inhibition in combination with FOLFIRINOX in patients with borderline resectable and locally advanced pancreatic cancer: a single-centre, open-label, dose-finding, nonrandomised, phase 1b trial. Lancet Oncol 2016;17:651-62.

16. Sanford DE, Belt BA, Panni RZ, et al. Inflammatory Monocyte Mobilization Decreases Patient Survival in Pancreatic Cancer: A Role for Targeting the CCL2/CCR2 Axis. Clin Cancer Res 2013;19:3404-15.

17. Iovanna JL, Closa D. Factors released by the tumor far microenvironment are decisive for pancreatic adenocarcinoma development and progression. Oncoimmunology 2017;6:e1358840.

18. Khagi Y, Kurzrock R, Patel SP. Next generation predictive biomarkers for immune checkpoint inhibition. Cancer Metastasis Rev 2017;36:179-90.

19. Conroy JM, Pabla S, Glenn ST, et al. Analytical Validation of a Next-Generation Sequencing Assay to Monitor Immune Responses in Solid Tumors. J Mol Diagn 2018;20:95-109.

20. Chaudhary R, Bishop J, Broomer A, et al. Estimating tumor mutation burden using next-generation sequencing assay. J Clin Oncol 2017;35:e14529.

21. Yan L, Zou L, Zhao W, et al. Clinicopathological significance of c-KIT mutation in gastrointestinal stromal tumors: a systematic review and meta-analysis. Sci Rep 2015;5:13718.

22. Dako. PD-L1 IHC 22C3 pharmDx - Rx Only - SK006 50 tests for use with Autostainer Link 48. 2019;1-38.

23. Dako. PD-L1 IHC 28-8 pharmDx - Interpretation Manual (Non-Squamous Non-Small Cell Lung Cancer) Dako Education Guides. Santa Clara, CA: Dako, 2016.

24. Shen H, Morrison CD, Zhang J, et al. 6p22.3 amplification as a biomarker and potential therapeutic target of advanced stage bladder cancer. Oncotarget 2013;4:2124-34.

25. Singh K, Tantravahi U, Lomme MM, et al. Updated 2013 College of American Pathologists/American Society of Clinical Oncology (CAP/ASCO) guideline recommendations for human epidermal growth factor receptor 2 (HER2) fluorescent in situ hybridization (FISH) testing increase HER2 positive and HER2 e. Breast Cancer Res Treat 2016;157:405-11.

26. Chen DS, Mellman I. Elements of cancer immunity and the cancer-immune set point. Nature 2017;541:321-30.

27. Conroy JM, Pabla S, Nesline MK, et al. Next generation sequencing of PD-L1 for predicting response to immune checkpoint inhibitors. J Immunother Cancer 2019;7:18. 
28. Woo S-R, Turnis ME, Goldberg MV, et al. Immune Inhibitory Molecules LAG-3 and PD-1 Synergistically Regulate T-cell Function to Promote Tumoral Immune Escape. Cancer Res 2012;72:917-27.

29. Okudaira K, Hokari R, Tsuzuki Y, et al. Blockade of B7$\mathrm{H} 1$ or B7-DC induces an anti-tumor effect in a mouse pancreatic cancer model. Int J Oncol 2009;35:741-9.

30. Ascierto PA, Bono P, Bhatia S, et al. LBA18Efficacy of BMS-986016, a monoclonal antibody that targets lymphocyte activation gene-3 (LAG-3), in combination with nivolumab in pts with melanoma who progressed during prior anti-PD-1/PD-L1 therapy (mel prior IO) in all-comer and biomarker-enriched. Ann Oncol 2017;28:v611-2.

31. Byers JT, Paniccia A, Kaplan J, et al. Expression of the Novel Costimulatory Molecule B7-H5 in Pancreatic Cancer. Ann Surg Oncol 2015;22:S1574-9.

32. Pedroza-Gonzalez A, Zhou G, Singh SP, et al. GITR engagement in combination with CTLA-4 blockade completely abrogates immunosuppression mediated by human liver tumor-derived regulatory $\mathrm{T}$ cells ex vivo. Oncoimmunology 2015;4:e1051297.

33. He LZ, Prostak N, Thomas LJ, et al. Agonist Anti-Human CD27 Monoclonal Antibody Induces T Cell Activation and Tumor Immunity in Human CD27-Transgenic Mice. J Immunol 2013;191:4174-83.

34. Akhmetzyanova I, Zelinskyy G, Littwitz-Salomon E, et al. CD137 Agonist Therapy Can Reprogram Regulatory T Cells into Cytotoxic CD4 + T Cells with Antitumor Activity. J Immunol 2016;196:484-92.

35. Piconese S, Valzasina B, Colombo MP. OX40 triggering blocks suppression by regulatory $\mathrm{T}$ cells and facilitates tumor rejection. J Exp Med 2008;205:825-39.

36. Schupp J, Krebs FK, Zimmer N, et al. Targeting myeloid cells in the tumor sustaining microenvironment. Cell Immunol 2019;343:103713.

37. Cannarile MA, Weisser M, Jacob W, et al. Colonystimulating factor 1 receptor (CSF1R) inhibitors in cancer therapy. J Immunother Cancer 2017;5:53.

38. Horenstein AL, Quarona V, Toscani D, et al. Adenosine Generated in the Bone Marrow Niche Through a CD38Mediated Pathway Correlates with Progression of Human Myeloma. Mol Med. 2016;22:694-704.

39. Cekic C, Day YJ, Sag D, et al. Myeloid Expression of Adenosine A2A Receptor Suppresses T and NK Cell Responses in the Solid Tumor Microenvironment. Cancer Res 2014;74:7250-9.

40. Bailey P, Chang DK, Nones K, et al. Genomic analyses identify molecular subtypes of pancreatic cancer. Nature
2016;531:47-52.

41. Cancer Genome Atlas Research Network. Electronic address: andrew_aguirre@dfci.harvard.edu; Cancer Genome Atlas Research Network. Integrated Genomic Characterization of Pancreatic Ductal Adenocarcinoma. Cancer Cell 2017;32:185-203.e13.

42. Weiss GJ, Blaydorn L, Beck J, et al. Phase Ib/II study of gemcitabine, nab-paclitaxel, and pembrolizumab in metastatic pancreatic adenocarcinoma. Invest New Drugs 2018;36:96-102.

43. Xue CB, Wang A, Han Q, et al. Discovery of INCB8761/ PF-4136309, a Potent, Selective, and Orally Bioavailable CCR2 Antagonist. ACS Med Chem Lett 2011;2:913-8.

44. Conroy T, Desseigne F, Ychou M, et al. FOLFIRINOX versus Gemcitabine for Metastatic Pancreatic Cancer. N Engl J Med 2011;364:1817-25.

45. Hutchinson L. Disrupting the chemokine axis in PDAC. Nat Rev Clin Oncol 2016;13:330.

46. Melisi D, Garcia-Carbonero R, Macarulla T, et al. Galunisertib plus gemcitabine vs. gemcitabine for first-line treatment of patients with unresectable pancreatic cancer. Br J Cancer 2018;119:1208-14.

47. Antonioli L, Pacher P, Vizi ES, et al. CD39 and CD73 in immunity and inflammation. Trends Mol Med 2013;19:355-67.

48. Haun RS, Quick CM, Siegel ER, et al. Bioorthogonal labeling cell-surface proteins expressed in pancreatic cancer cells to identify potential diagnostic/therapeutic biomarkers. Cancer Biol Ther 2015;16:1557-65.

49. Blando J, Sharma A, Higa MG, et al. Comparison of immune infiltrates in melanoma and pancreatic cancer highlights VISTA as a potential target in pancreatic cancer. Proc Natl Acad Sci U S A 2019;116:1692-7.

50. Moffitt RA, Marayati R, Flate EL, et al. Virtual microdissection identifies distinct tumor- and stromaspecific subtypes of pancreatic ductal adenocarcinoma. Nat Genet 2015;47:1168-78.

51. Collisson EA, Sadanandam A, Olson P, et al. Subtypes of pancreatic ductal adenocarcinoma and their differing responses to therapy. Nat Med 2011;17:500-3.

Cite this article as: Lenzo FL, Kato S, Pabla S, DePietro P, Nesline MK, Conroy JM, Burgher B, Glenn ST, Kuvshinoff B, Kurzrock R, Morrison C. Immune profiling and immunotherapeutic targets in pancreatic cancer. Ann Transl Med 2021;9(2):119. doi: 10.21037/atm-20-1076 